less than the risk of respiratory failure. Thus, single LT may be chosen for cases with low bleeding risk, such as COPD. However, some measures should be taken for double LT: decrease the time on the waiting list (emergency code), increase the pretransplant hemoglobin (erythropoietin), and consider preoperative self-donation. Multiple transfusions can be required given the marrow-suppressing effect of the medications and anemia of chronic disease. These were not present in our case, but they could be treated with erythropoietin and pharmacologic efforts.

From the beginning of our LT program in October of 1993, we have performed approximately 400 LTs. In the last 10 years, 70 LTs were for COPD, and the patients remained in the ICU for 12 days and the hospital for 45 days. The described case was within normal ranges, and these results may be due to Spain's social and health services system.

\section{CONCLUSIONS}

Our case showed that LT can be performed safely in Jehovah's Witnesses and offers a new perspective when deciding whether to perform major surgery and at the same time comply with the conditions that Jehovah's Witnesses follow, allowing for LT to be performed in patients who otherwise would have been excluded from the list of candidates.

\section{References}

1. Elmistekawy E, Mesana TG, Ruel M. Should Jehovah's Witness patients be listed for heart transplantation? Interact Cardiovasc Thorac Surg. 2012;15:716-9.

2. Conte JV, Orens JB. Lung transplantation in a Jehovah's Witness. J Heart Lung Transplant. 1999;18:796-800.

3. Grande AM, Rinaldi M, D'Armini AM, Pellegrini C, Viganò M. Lung transplantation in a Jehovah's witness. Case report in a twinning procedure. J Cardiovasc Surg (Torino). 2003;44:131-4.

4. Beauchamp TL. Methods and principles in biomedical ethics. J Med Ethics. 2003 29:269-74

5. Mallory GB Jr. Challenging issues associated with organ transplantation for Jehovah's Witness individuals. J Heart Lung Transplant. 2000;19:119-20.

\title{
Acute bilateral pulmonary embolism and complete occlusion of the left internal carotid artery caused by paradoxical embolus through a persistent foramen ovale in a 32-year-old woman
}

\author{
Brigitte Gansera, MD, ${ }^{\mathrm{a}}$ Michael Bauer, MD, ${ }^{\mathrm{b}}$ Laura Gansera, ${ }^{\mathrm{a}}$ and Walter Eichinger, MD, PhD, ${ }^{\mathrm{a}}$ Munich, \\ Germany
}

In 1877 , Cohnheim ${ }^{1}$ was the first to report a case of paradoxical embolism. $\mathrm{Zahn}^{2}$ described the first patient in which necropsy revealed a thrombus localized in the foramen ovale. Cerebrovascular accidents caused by paradoxical embolism through a persistent foramen ovale are rare, but have been described as a reason for them, especially in young patients with no other risk factors. ${ }^{3}$ We describe, to our knowledge, a unique case of a 32year-old woman with pulmonary embolism and occlusion of the left internal and external carotid arteries caused by paradoxical embolism through a patent foramen ovale (PFO).

\footnotetext{
From the Department of Cardiovascular Surgery ${ }^{\mathrm{a}}$ and the Department of Radiology, ${ }^{\mathrm{b}}$ Klinikum Bogenhausen, Munich, Germany.

Disclosures: Authors have nothing to disclose with regard to commercial support.

Received for publication Jan 13, 2013; revisions received Feb 10, 2013; accepted for publication Feb 27, 2013; available ahead of print March 26, 2013.

Address for reprints: Brigitte Gansera, MD, Department of Cardiovascular Surgery, Clinic Bogenhausen, Munich, Englschalkingerstr. 77, 81925 Munich, Germany (E-mail: brigitte_gansera@web.de).

J Thorac Cardiovasc Surg 2013;145:e67-9

$0022-5223 / \$ 36.00$

Copyright (C) 2013 by The American Association for Thoracic Surgery

http://dx.doi.org/10.1016/j.jtcvs.2013.02.054
}

\section{CLINICAL SUMMARY}

A 32-year-old woman with clinical signs of right-side hemiplegia, aphasia, and dyspnea was transferred urgently from the stroke unit of another hospital to our department of cardiac surgery. The day before, the patient experienced an isolated, sudden occurrence of right-hand paresthesia and dizziness. She also complained of left-side leg pain, without other previous symptoms. Risk factors for deep venous thrombosis were oral contraceptives, smoking, and extreme obesity (body mass index, 50).

Computed tomography (CT)-angiography was performed and sagittal sinus thrombosis was detected. Duplex ultrasound of the extremities showed thrombosis of the right and left femoral veins. Initially, the patient received a bolus of $5000 \mathrm{IU}$ heparin followed by continuous administration of heparin (30,000 IU/24 hours). The next day the patient's neurologic symptoms worsened rapidly (right-side hemiplegia and aphasia) and were accompanied by dyspnea.

Additional duplex ultrasound and CT-angiography revealed complete occlusion of the left internal carotid artery (Figure 1); the vertebral arteries were normal. Transcranial ultrasound showed collateralization via the communicating artery from the right side to the left. At first, cerebral CT did not show any pathologic results. With the suspicion of 


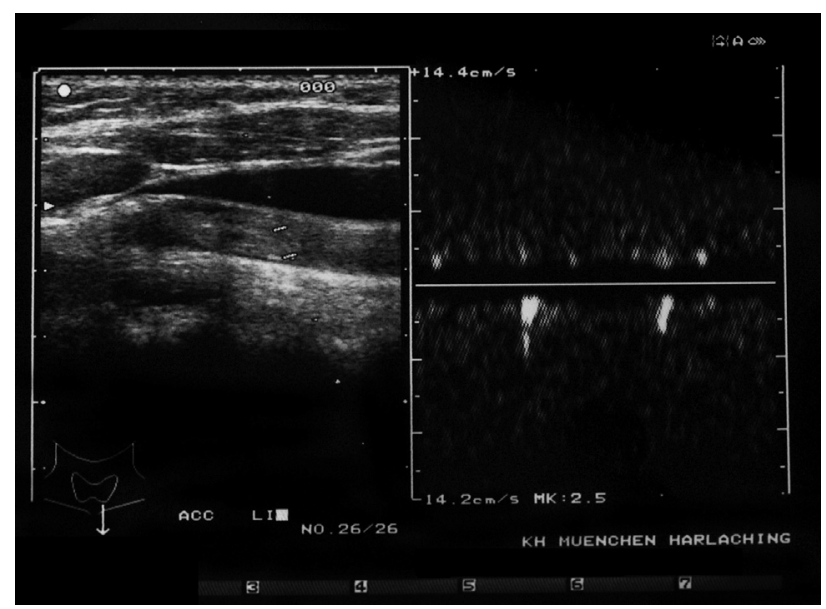

FIGURE 1. Duplex ultrasound shows thrombosis of the left carotid artery.

pulmonary embolism, spiral CT of the chest was performed and it showed multiple, huge embolizations of the central left and right pulmonary arteries.

A predominant atrial left-to-right shunt was detected via "outwash phenomenon" by transthoracic contrast echocardiography. Transesophageal echocardiography revealed a large PFO without thrombotic material protruding through the PFO, as well as a sporadic pass-over of "bubbles" from the right to left atrium, which indicates a crossed shunt.

Severe tricuspid regurgitation and a dilated right ventricle were observed; pulmonary artery systolic pressure was estimated to be $75 \mathrm{~mm} \mathrm{Hg}$ by measuring tricuspid regurgitant velocity. The patient's platelet count did not drop lower than 180/nL. Although typical onset of heparin-induced thrombocytopenia (HIT) occurs 5 to 10 days after the institution of therapy, several assays for the diagnosis of HIT were used to estimate the probability of HIT for avoidance of an additive exposure and risk for the patient after heparinization during the use of extracorporeal circulation. Heparin-induced thrombocytopenia type I was negative, as was the clinical scoring system (4Ts score: thrombocytopenia, time of platelet count fall, thrombosis or other sequelae, and other causes for thrombocytopenia) and the test for platelet factor 4/heparin antibodies (enzyme-linked immunosorbent assay) for HIT type II. With the awareness that there was a risk of converting the ischemic stroke to a hemorrhagic one, the decision for immediate surgery was based on the acute nature and worsening of neurologic symptoms, and on the absence of infarction-marked zones detected via cerebral CT.

During emergency surgery, we first performed a thromboendarterectomy of the left carotid artery and removed a $4 \times 1-\mathrm{cm}$ thrombus from the internal carotid artery (Figure 2). Then, after installation of cardiopulmonary bypass under moderate hypothermia, we performed an embolectomy of both pulmonary arteries and finally closed the PFO with single interrupted sutures. Evaluation for

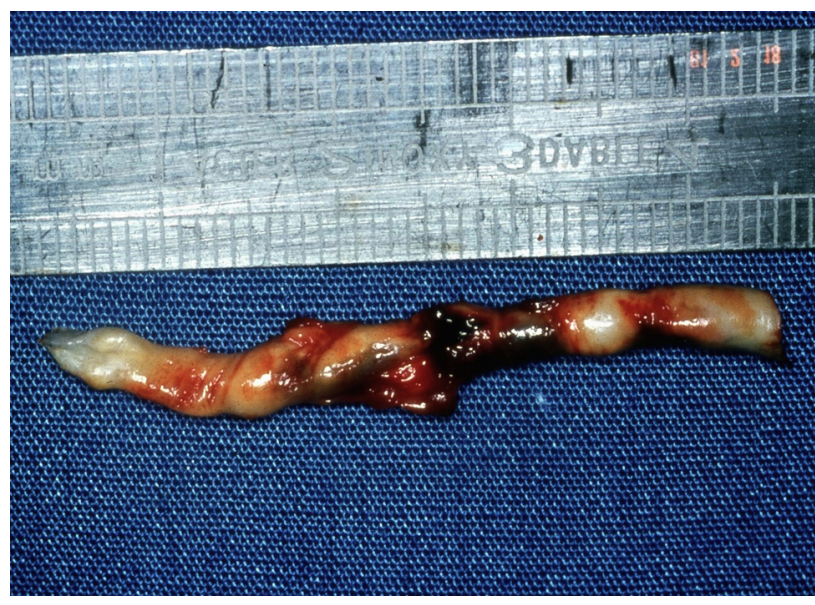

FIGURE 2. The surgical specimen in the left internal carotid artery.

a hypercoagulable status (including factor II and factor V Leiden) was negative, and borreliosis serology as well as protein $\mathrm{C}$ values were within the normal range. The postoperative course was uneventful and the patient was extubated 7 hours after surgery. Two days postoperatively, neurologic symptoms (aphasia and hemiplegia) were completely reversed, although a control of the cerebral $\mathrm{CT}$ showed left-side infarction zones in the area of the middle cerebral artery, the prefrontal artery, the pre-Rolandic artery, the angular gyrus artery, the insulary artery, and the temporooccipital artery. The patient was discharged on postoperative day 9 for rehabilitation. Warfarin anticoagulation therapy was recommended for 6 months.

Three years postoperatively, the patient became pregnant with twins. Gravidity and childbirth proceeded without complications, and the patient did not sustain any residual neurologic symptoms. ${ }^{3}$ A follow-up 11 years after surgery indicated that the patient is in good condition, apart from extreme adiposity (body mass index, 55).

\section{DISCUSSION}

A PFO is described to be present in $10 \%$ to $25 \%$ of normal human hearts. ${ }^{4,5}$ Pulmonary hypertension, primarily a result of pulmonary embolism, is a common predictor for paradoxical embolism. Most underlying disease is deep vein thrombosis of the lower extremities. Transthoracic and transesophageal echocardiography are the main diagnostic tools, followed by CT and ultrasound of the lower extremities. Although cerebrovascular accidents are noted to occur in the presence of PFO, complete acute occlusion of the internal carotid artery seems to be a very rare complication and indicates emergency surgery if worsening neurologic symptoms are present.

\section{References}

1. Cohnheim J. Thrombose und Embolie. Vorlesungen Allgemeine Pathologie. 1877; $1: 143-4$. 
2. Zahn FW. Untersuchungen über Thrombose. Virchowas Arch Pathol Anat. 1899;115:71.

3. Mas JL, Arquizan C, Lamy C, Zuber M, Cabanes L, Derumeaux G, et al. Recurrent cerebrovascular events associated with patent foramen ovale, atrial septal aneurysm, or both. $N$ Engl J Med. 2001;345:1740-6.
4. Loscalzo J. Paradoxical embolism: clinical presentation, diagnostic strategies, and therapeutic options. Am Heart J. 1987;114:141-5.

5. Mas JL. Diagnosis and management of paradoxical embolism and patent foramen ovale. Curr Opin Cardiol. 1996;11:519-24.

\title{
Ventricular assist device abdominal driveline infection: Treatment with platelet-rich plasma
}

\author{
Federica Jiritano, MD, Giuseppe Filiberto Serraino, MD, Lucia Cristodoro, MD, and \\ Attilio Renzulli, MD, PhD, Catanzaro, Italy
}

The contemporary trend toward left ventricular assist device (LVAD) implantation as a definitive treatment for end-stage heart failure has yielded a valid alternative for those patients who are not suitable candidates for heart transplantation and whose conditions are refractory to medical treatment. ${ }^{1}$ The long-term use of such devices, however, has brought into the spotlight a number of complications related to the interaction between the human body and the mechanical component. One of these is driveline infection, which in the worst-case scenario could lead to sepsis, forced explantation of the LVAD, or even death. ${ }^{2}$ The treatment is challenging and sometimes delayed because the problem occurs in patients already out in in the community. Vacuum-assisted closure (VAC) therapy, targeted antimicrobial therapy, and other advanced dressings have been proposed as valid treatments for driveline infections. ${ }^{3}$ We report 2 cases in which the previously mentioned measures failed to cure the infection, whereas continued topical application of autologous platelet-rich plasma (PRP) over the wound for about 2 weeks resolved the infection and closed the exit wound.

\section{CLINICAL SUMMARIES}

\section{Patient 1}

A 49-year-old man with end-stage cardiac failure (New York Heart Association functional class IV) was admitted with ascites and orthopnea. Five days after the hospital admission, the patient had acute abdominal pain, proctorrhagia, and anemia, necessitating urgent laparotomy.

\footnotetext{
From the Cardiac Surgery Unit, Magna Græcia University of Catanzaro, Catanzaro, Italy.

Disclosures: Authors have nothing to disclose with regard to commercial support. Received for publication Jan 24, 2013; revisions received Feb 9, 2013; accepted for publication Feb 28, 2013; available ahead of print March 25, 2013.

Address for reprints: Federica Jiritano, MD, Cardiac Surgery Unit, Magna Græcia University of Catanzaro, Catanzaro 88100, Italy (E-mail: fede.j@hotmail.it). J Thorac Cardiovasc Surg 2013;145:e69-70 $0022-5223 / \$ 36.00$

Copyright (c) 2013 by The American Association for Thoracic Surgery http://dx.doi.org/10.1016/j.jtcvs.2013.02.072
}

Ischemic colitis involving the rectum was found; it was resected, and a left colostomy was performed. Hemodynamic conditions improved steadily, and the patient was extubated on the third postoperative day. However he developed renal failure requiring hemodialysis. After 10 days, the HeartWare VAD system (HeartWare International, Inc, Framingham, Mass) was implanted. Seven days later, the skin around the exit site of the abdominal cable became erythematous with serum and purulent discharge. Escherichia coli was isolated, and intravenous treatment with ciprofloxacin was started on the basis of the antibiogram. After 1 week of antibiotic therapy, the infection had not improved. The exit site wound was then dressed every 3 days for a total of 5 times with autologous PRP until the 28th postoperative day, when it appeared completely closed with good tissue healing. Chronic kidney failure necessitating daily dialysis persisted. On the 32 nd postoperative day, the patient had acute cardiovascular and respiratory failure develop, necessitating mechanical ventilation and maximum inotropic support. Cardiac tamponade was diagnosed, and $1.5 \mathrm{~L}$ of bloodstained fluid was drained. The patient's cardiac function recovered, but he could not be weaned from mechanical ventilation and dialysis. He died on the 50th postoperative day of multiple organ failure. No signs of skin infection were found around the driveline.

\section{Patient 2}

A 50-year-old man who was obese (body mass index 36 $\mathrm{kg} / \mathrm{m}^{2}$ ) and had diabetes and dilated cardiomyopathy was admitted to hospital for end-stage cardiac failure. The patient underwent LVAD (HeartWare) implantation through a median sternotomy. His postoperative course was uneventful, and he was discharged on the 15 th postoperative day. On the 30th postoperative day, a purulent and smelly discharge developed at the abdominal cable exit site. Swabs were sent for culture, and multiresistant Acinetobacter baumannii (sensitive to colistimethate sodium [INN colistin]) and Staphylococcus aureus (sensitive to linezolid) were isolated. Intravenous antibiotic treatment targeted 